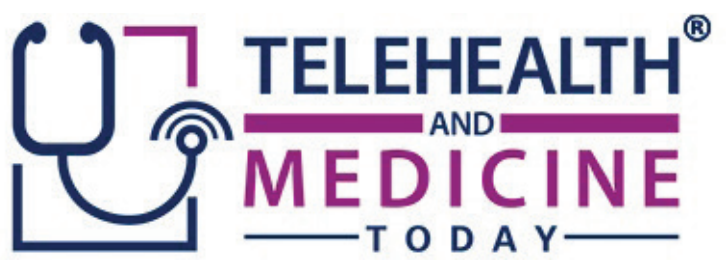

\title{
Patient- and Family-Centered Video Rounds in the Pediatric Intensive Care Unit
}

\author{
Ryan L. DeSanti, Diane H. Brown, Sushant Srinivasan, Tom Brazelton, \\ Michael Wilhelm
}

Affiliations: Department of Pediatrics, University of Wisconsin School of Medicine and Public Health, Madison, WI, USA

Corresponding Author: Michael Wilhelm, Email: mwilhelm@pediatrics.wisc.edu

Keywords: Coronavirus, Patient-centered Rounds, Socially Distanced Rounds, Telemedicine

Section: Proof of concept

Objective: Management of the coronavirus disease 2019 (COVID-19) pandemic has required social distancing requirements and personal protective equipment shortages, which have forced hospitals to modify patient care rounds. We describe our process developing telemedicine rounds to maintain synchronous, multidisciplinary, pediatric intensive care unit rounds. By adapting available resources using rapid process improvement (PI), we were able to develop patient- and family-centered video rounds (PFCVR).

Design: When rounding team members were forced to work from home, we adapted an existing telemedicine platform (VidyoConnect) to perform PFCVR. A quality improvement (QI) team developed an initial standard process, which underwent rapid PI using a small multidisciplinary team.
Setting: A 21-bed, mixed medical/surgical/ cardiac pediatric intensive care unit.

Participants: Critical care patients, families, physicians, consultants, nurses, and ancillary staff.

Interventions: The QI team initially met daily, then weekly, sought feedback from nurses, families, and other care providers, and utilized small tests of change to improve the rounding process.

Results: We established standardized, socially distanced rounds using VidyoConnect to allow synchronous, multidisciplinary PFCVR. Implementation of a schedule and rounding script facilitated efficient and effective team communication, optimized participation by the entire team, and decreased interruptions. 
Conclusions: The COVID-19 pandemic compromised the feasibility of the previous rounding process. PFCVR is a safe and effective tool to facilitate communication while adhering to social distancing guidelines. Use of available platforms and team-based PI is critical for successful implementation.

\section{BACKGROUND}

The pandemic caused by coronavirus disease 2019 (COVID-19) required hospitals to rapidly change workflows to prevent the spread of infection, including to hospital staff. The Centers for Disease Control and Prevention recommends maintaining at least 6-ft. distance between people (social distancing) and limiting contact with persons outside the immediate household. ${ }^{1}$ This, combined with escalating healthcare utilization and limited personal protective equipment, led hospitals to enforce stay-at-home orders for many providers normally involved in patient care. Pharmacists, dieticians, child life, language translators, and physicians (residents, fellows, and faculty) were asked to work remotely and limit bedside staff to essential hands-on caregivers, often a nurse and respiratory therapist (RT). Teams have further been asked to limit the number of providers performing patient evaluations, limiting exams to only those necessary and for emergent care.

When the State Department of Health instituted stay-at-home orders on March 25, hospital's university hospital, community hospital, and children's hospital soon after issued restrictions on the presence of essential personnel not providing direct patient care. ${ }^{2}$ In the children's hospital's pediatric intensive care unit (PICU), a 21-bed, mixed medical/surgical/cardiac unit, daily patient- and family-centered rounds (PFCR) followed a traditional format, with large multidisciplinary teams at the patient's bedside, discussing the daily plan of care with the bedside nurse and parents. PFCR had been adapted to enhance family involvement and understanding, provide trainee role modeling and enhanced education, and improve team communication. ${ }^{3}$ However, given the restrictions induced by COVID-19, a new format for rounds was necessary to continue PFCR, to maintain its associated benefits.

We describe herein how we developed a novel, telemedicine-based approach to maintain social distancing while including the entire team in synchronous patient- and family-centered video rounds (PFCVR). The objective of this manuscript is to describe the process used to adapt our existing telemedicine resources for telerounding, and to discuss our rapid process improvement (PI) and management of ongoing challenges as an example for other programs that wish to implement PFCVR.

\section{METHODS}

This project was determined to constitute quality improvement by the hospital Institutional Review Board (IRB) and was not subject to full IRB review.

\section{Pre-COVID-19 Rounding Structure}

Our PICU utilized a two-team rounding model involving a "cardiovascular services team" (cardiac team) and a "medical-surgical team" (medical team) that rounded simultaneously. The cardiac team included the family, bedside nurse and fellows, nurse practitioners (NP)/physician assistants, and faculty physicians from PICU, cardiology, and cardiac surgery. The medical team included the family, bedside nurse, two resident physicians, a PICU fellow, and faculty physician. Pediatric pharmacists, dieticians, and RTs moved between the teams based on individual patient's needs. Subspecialists would join rounds depending on their availability and involvement with a patient's care, or would 
discuss their recommendations with the team outside of rounds. Cardiology and cardiac surgery presence on rounds were consistent on the cardiac team.

\section{Telemedicine Capabilities and PICU Resources}

VidyoConnect (Vidyo Inc, Hackensack, NJ), a web-based, Health Insurance Portability and Accountability Act (HIPAA)-compliant, video conferencing (VC) system, was in use at hospital, primarily for remote outpatient visits. Prior to COVID-19, PICU providers had rarely used VC for remote consultation during medical control of regional transports and none had used VidyoConnect to provide inpatient care. The PICU was pre-equipped with wireless internet access and 23 iPads (Apple Inc, Cupertino, CA), used for parental access of MyChart Bedside (Epic Systems Corporation, Verona, WI) to view electronic health record (EHR) information. No other specific telehealth resources were initially available.

\section{COVID-19 Staffing and Rounds}

On March 17, hospital began mandatory work-from-home policies for consultants and ancillary staff. Over the next 14 days, institutional restrictions continued to increase, with harmful effects on collaboration and patient care. PFCR were significantly impacted, as typically more than 10 participants would be involved in rounds on one patient. This would have required over $360 \mathrm{ft}^{2}$ of space for rounds to occur and maintain social distancing. The PICU therefore decided to trial remote telerounding to restore synchronous, multidisciplinary collaboration and discussions, while continuing to minimize exposure to the patient care environment and one another.

Family member visitation was also restricted during this time to two family members per patient. Parents were only allowed in the patient's room and in the hallway, when leaving the hospital. Patient rooms have accommodating pullout beds and private bathrooms for family that spend the night. Family was restricted from all other hospital common spaces. Family members were unable to access the VC system remotely, as it requires a hospital-issued EHR login and password.

\section{RESULTS}

\section{Implementation of Remote Rounding}

Bedside nurses logged into VidyoConnect using unit iPads and participated in rounds with the family in the patient's room. All other team members and consultants for each patient in the PICU were directly shown how to install and use VidyoConnect by the PICU team during the initial trial. These instructions were then disseminated to all of American Family Children's Hospital (AFCH) in a written form (Addendum 1a-c). Remote rounding was first trialed on March 31 (Figure 1). Each PICU team rounded in one virtual "room," where nurses (with families), consultants, and other team members would join. This immediately restored synchronous rounds while strictly adhering to social distancing.

The team initially followed the same rounding structure that existed prior to staffing restrictions, enabling participation by physically distanced team members and consulting teams. However, a number of challenges existed while using $\mathrm{VC}$, which were more noticeable than when the entire team was in one physical space. Three main issues were identified: poor audio-visual quality, lack of availability for rounds when the rest of the team was ready, and lack of consistent opportunities to contribute to rounds. We therefore chose to implement a number of PI projects using lean process management tools to improve the 


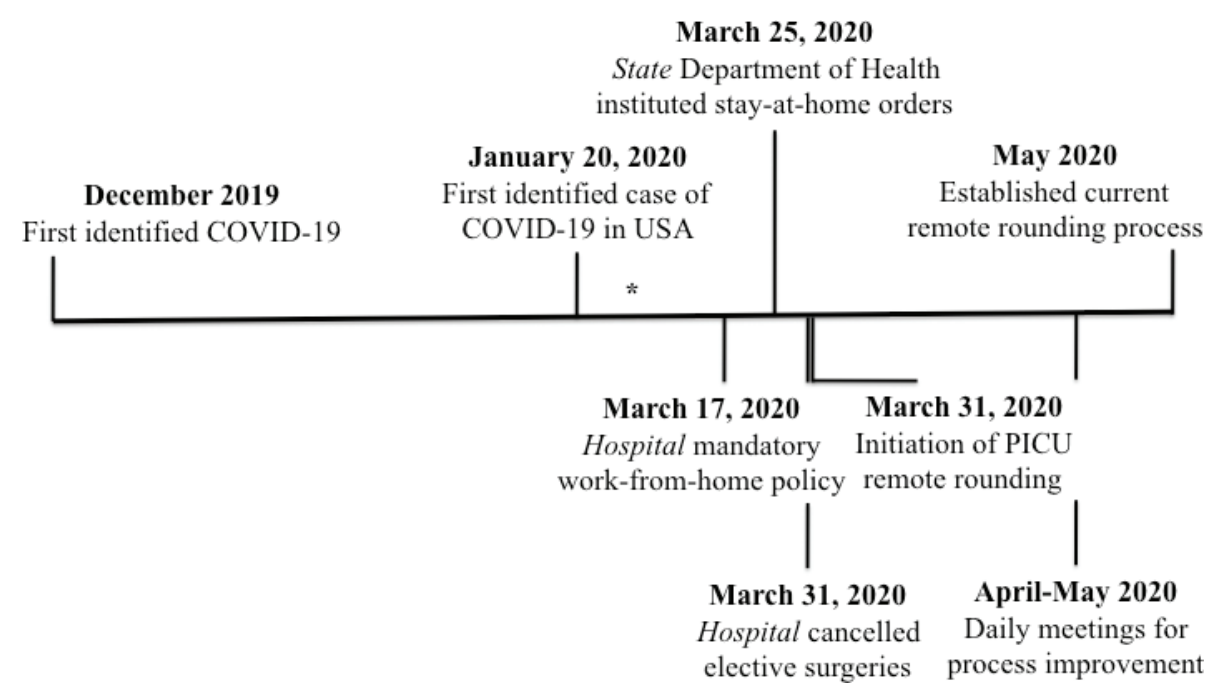

Figure 1-Timeline description of pediatric intensive care unit remote rounding process. *First COVID-19 case reported in State, February 5, 2020.

process for providers, families, and members of the multidisciplinary team.

\section{Quality Assurance and Process Improvement}

A PI team, consisting of five PICU physicians (three faculty and two fellows), a dietician, a pharmacist, the PICU nurse manager, and the PICU clinical nurse specialist, initially met daily and then weekly to discuss the current process and areas for improvement. Feedback was verbally solicited from rounding team members including family. Areas for improvement were identified by utilizing plando-study-act small tests of change cycles and problem solving. ${ }^{4-7}$ Identified problems and possible solutions were rapidly implemented and reviewed through this process.

Initial efforts targeted three issues: the audiovisual setup of the physician's rounding room, notification of the bedside nurse that the team was ready to round on their patient, and development of a rounding script to engage all team members. Small tests of change were performed daily with each of these processes until a new standard was created and added to the overall standard work.

\section{Standardized Remote Rounds}

Within 3 weeks, we established a standard remote rounding process allowing all team members to participate in synchronous PFCVR (Figure 2). A schedule was utilized to ensure that the correct team members were in the rounding "room" to discuss a specific patient. At our daily morning huddle, the team determined a rounding order and estimated time needed for each patient. Patients requiring subspecialist involvement were performed sequentially whenever possible. Our rapid PIs determined we needed 5-minutes for transition between patients to ensure the previous nurse and family had left the rounding room prior to starting the next patient, and to give the next nurse and family time join.

To communicate the rounding schedule to other team members, the charge nurse documents the assigned rounding start time, end time, and virtual "room" in the EHR 30 minutes prior to the start of rounds to allow team members to plan their mornings. Consulting services can request specific rounding times, prior to the huddle by contacting either the PICU charge nurse or the fellow and were required to log into the EHR to 


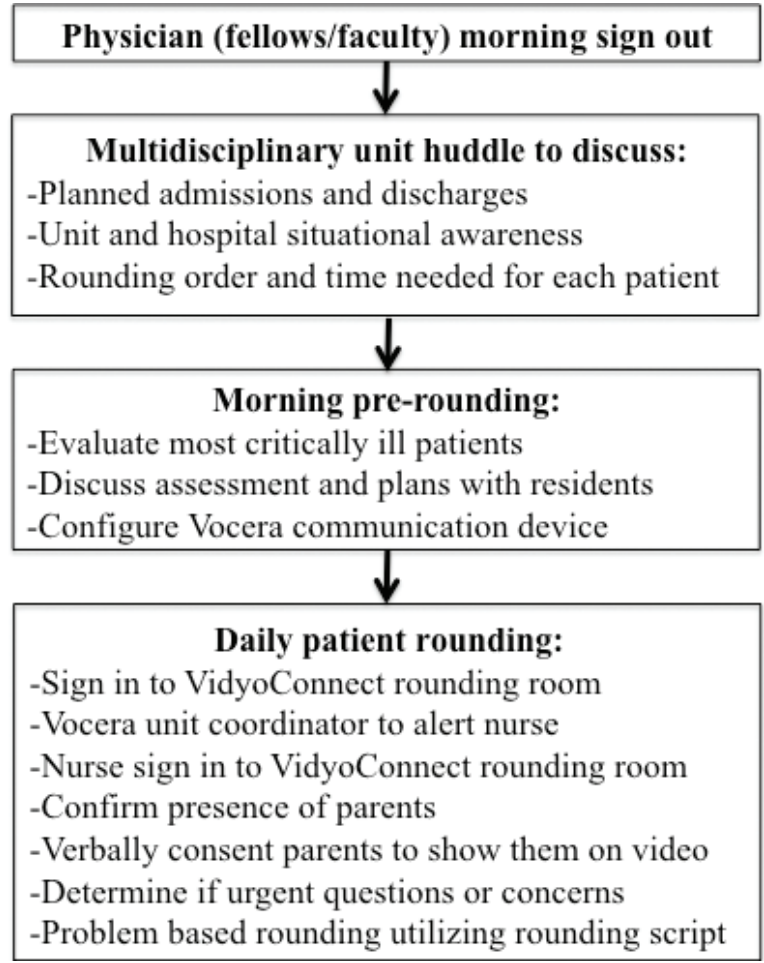

Figure 2-Final workflow for pediatric intensive care unit rounding process.

determine the rounding time for their patients. At the assigned time, team members log into the assigned patient rounding room. The PICU physicians (two residents, a fellow, and a faculty member) sit in a central conference room where VidyoConnect is displayed on a large television with VC capabilities (Figure 3). Nurses log-in to VidyoConnect on unit iPads in the patient's room with the family present; and remote team members log-in on their hospitalapproved devices.

A standard process for rounds (rounding script) was developed to ensure opportunities for each person to speak and minimize interruptions. The PICU fellow (or attending faculty) leads the rounding discussion, facilitating the presentation by the primary provider for the patient (i.e. resident or NP). After confirming nurse and family presence, the fellow introduces

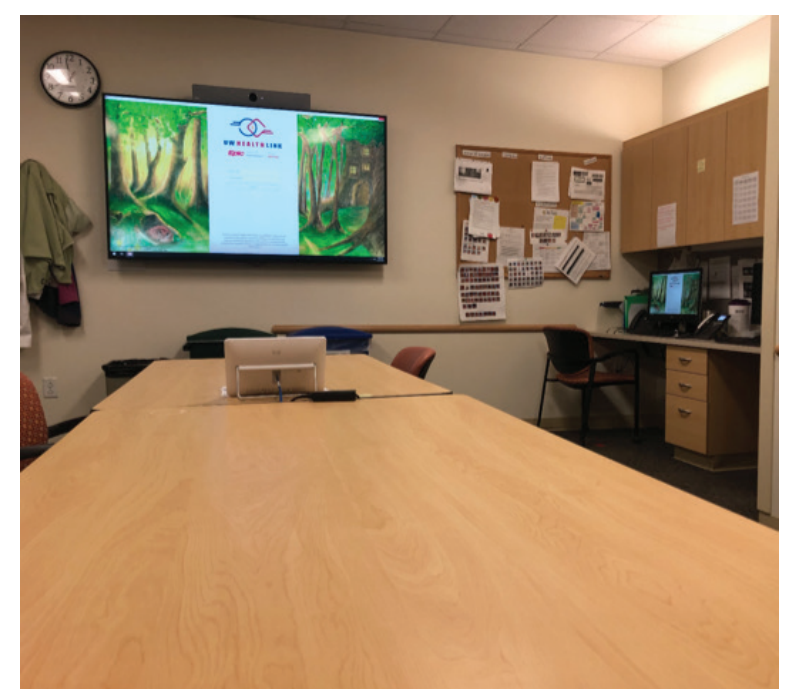

Figure 3-Picture of unit conference room.

the patient using a one-line summary statement, asks what problems/concerns the family and nurse would like addressed during rounds, and then identifies the most urgent PICU problem to be addressed that day using the rounding script and checklist (Figure 4, Addendum 2). The NP or resident then summarizes the current state of the problem and presents relevant data to support an assessment and plan. The nurse and/or RT share their current physical examination, continuous medication infusion rates, and respiratory support settings as appropriate. The PICU fellow ensures the presented information is complete, prompting for additional information as needed, and specifically asks for input from other team members. Because nonverbal cues indicating someone would like to speak are less apparent during VC, the PICU fellow specifically asks individual team members for their input, as appropriate. The resident or NP then verbalizes the plan and the fellow clarifies or corrects, if needed. After the team addresses the primary problem, the fellow then identifies the next most important problem and the process repeats. A second resident enters patient orders throughout the presentation, and order read-back is completed before moving to the next patient. 
The final major PI centered on transitions between patients, as the team was no longer physically moving between rooms. Vocera badges (Vocera Communications Inc, San Jose, CA) were used as a direct form of communication to alert the unit coordinator that the previous patients' rounds were complete and that he/she should alert the next scheduled nurse to join rounds.

\section{Clinical Examinations}

Stable patients were examined once before rounds by the resident or NP caring for them and once after (commonly in the afternoon to separate physician examinations as much as possible) by either the PICU faculty physician. For critically ill patients or unstable patients, the fellow and/or faculty physician would examine the patient as necessary for clinical decision-making. Bedside nurses would alert physicians to any change in clinical status prompting repeat examination.

\section{PFVCR Utilization}

From March 31 to April 27, the median PICU census was six patients per day (interquartile range (IQR) 6-8.25; mean 7) and involved 199 separate patient rounding events. The described PFVCR was utilized on all PICU patients during this period. A median of 22

1. Team lead (PICU fellow or supervising faculty), invites the team members and bedside nurse to join rounds When the RN joins rounds:

-RN shows patient to the team before the start of the presentation

-Team lead asks for family or nursing concerns to address during rounds

-Address concerns immediately or indicate when they'll be addressed

2. Team lead gives background about patient and overall summary statement

3. The team lead solicits data, assessment, and plan in problem-based manner

For each problem, the team lead prompts (if necessary) for:

-A trajectory

-Data to support assessment (vital signs, exam, labs, imaging, other necessary data)

The team lead may need to ask for specifics or to add data themselves

- Others (nursing, RT, pharmacy) to provide up-to-the minute data

Be sure to also solicit the impression of these team members and plans they'd like considered

- Input from the primary consultant(s) for that problem

Ideally each person is asked, by name, in turn

-Either a summary of the assessment and plan or if this needs to wait for discussion of a subsequent problem

4. Refer to the PICU Rounds Checklist to ensure all systems/problems covered

- Some problems should be discussed for all patients (HACs, nutrition, VTE)

- All medications should be for a specific problem, so make sure all are discussed

5. Team lead prompts for a summary of the assessment and plan

- Include lab schedule and lines/drains/tubes

6 Team lead asks the rest of the team if they have questions or comments

- Ideally they ask each person, in turn, by name

- Ask bedside nurse (and family), "What questions do you have?"

7. The team lead closes rounds on the patient

Figure 4-Pediatric intensive care unit rounding script.

$\mathrm{HAC}=$ hospital acquired conditions, $\mathrm{PICU}=$ pediatric intensive care unit, $\mathrm{RN}=$ registered nurse, $\mathrm{RT}=$ respiratory therapy,

$\mathrm{VTE}=$ venous thromboembolism 
providers/day utilized VidyoConnect for rounds in the PICU (IQR 18-25; mean 22); there were a median of two VC calls per provider/day (IQR 1-4; mean 3) lasting a median of 22 minutes per call (IQR 11-38 minutes; mean 41 minutes). All care providers who normally participate in PICU PFCR participated in PFVCR during the study period and most non-PICU sub-specialties participated. These included cardiology, pulmonology, neurology, nephrology, infectious disease, endocrinology, hematology, neonatology, cardiovascular surgery, neurosurgery, general surgery, plastic surgery, otolaryngology, complex care, and psychiatry. There were no providers who wished to participate in PFCVR who were excluded from rounding.

\section{DISCUSSION}

Access to telehealth services has increased substantially in the years prior to the current COVID-19 pandemic. ${ }^{8}$ Nearly three-fourths of hospital systems report having a computerized telehealth program, yet only $15 \%$ of pediatricians report using these technologies to provide patient care. ${ }^{8,9}$ Telemedicine remains primarily employed for outpatient and outreach services in rural practices and in the western United States. ${ }^{8,10}$ The pandemic rapidly changed the telemedicine landscape and many barriers to widespread implementation, including discomfort of patients, parents, and providers; low reimbursement rates; federal privacy restrictions; and preference for in-person visits. ${ }^{10,11}$

Despite the ongoing need for social distancing to decrease the spread of infection, there remain few published examples of inpatient services utilizing remote telehealth rounding. ${ }^{12-15}$ Telehealth is also being used increasingly for consultative services ${ }^{16,17}$ and to enhance communication between providers in specific circumstances. ${ }^{18-21}$ PFCRs in the PICU typically involve more than 10 people, including the patient and family, requiring over $360 \mathrm{ft}^{2}$ of space to achieve safe social distancing. Yet benefits of PFCR are clearly established (3, 22), we therefore sought to establish a method to minimize exposure of the team and patients, while continuing our PICU "bedside" rounds with the same breadth of contributing team members.

Many reports have focused on the need for a robust system-wide support structure to maintain viability of telehealth services. ${ }^{23}$ While our institution has such system-wide infrastructure, resources during the early pandemic were primarily focused on outpatient and regional care given in our rural catchment area. We herein described our process to rapidly develop an inpatient remote rounding, telehealth program using minimal system-wide resources and an already available HIPAA-compliant VC system (Table 1).

Our program was built by frontline providers from a readily available VC system utilizing existing infrastructure (wireless internet and iPads) within the PICU. Similar to the report from Stanford Children's Health, we adapted an existing technology with a goal of rapid deployment with minimal training of parents and personnel to maintain our robust multidisciplinary, family-centered rounding process. ${ }^{12}$ The initial implementation highlighted many of the communication problems we previously experienced with the in-person rounding and brought additional challenges. By soliciting team feedback and employing rapid PI strategies, we were able to overcome many of these concerns and implement a robust program that was beneficial for all team members. 
Table 1: Steps to establish remote rounding. $U C=$ unit coordinator

\section{Pre-established infrastructure \\ 1. Hospital-wide wireless internet access \\ 2. Video conferencing system \\ 3. 23 PICU iPads available}

\section{Steps to establish round infrastructure}

1. Install video conferencing system on iPads

2. Purchase a tablet mounting stand

3. Educate and prepare rounding team for remote rounding implementation

4. Trial remote rounding

Identified rounding challenges

1. Unclear communication and difficulty hearing primary presenters

2. Frequent presenter interruptions

3. Establishment of correct team at correct rounding time

4. Communication with nurse the team is ready for rounds

\section{Quality assurance \& process improvement}

1. Identify team member

2. Verbally solicit feedback from rounding team and family members

3. PDSA cycles and small tests of change

\section{Attempted solutions}

1. Re-configure seating arrangement in physician room; purchase speaker; move conferencing system to central location

2. Establish rounding script with prompting of consulting team members

3. Rounding schedule with prescribed duration of rounding length; education of consulting teams

4. Communication device to alert UC team prepared for patient, UC to alert nurse

PDSA = Plan-Do-Study-Act, PICU = pediatric intensive care unit.

Our previous rounding process lacked consistency between attending providers both with regard to the process for individual patients, and the duration and order for rounds as a whole. In order to facilitate participation by each team member, we developed a rounding script for the presenting resident, fellow, or NP. This script included prompts for input from each team member, focusing on their areas of expertise (i.e. dietician for meals and weight gain), up to date physical examination, continuous medication infusion rates, respiratory support settings from bedside staff; and codified asking parents and the nurse for specific concerns at the start of each patient's rounds. Other necessary changes included formally introducing each team member, which often went unnoticed during traditional, in-person rounds. Finally, our PICU had attempted for many years to implement a schedule, as described in other PICUs, to ensure families and other team members could be present for rounds. ${ }^{24}$ However, with the need to coordinate multiple providers entering the telemedicine "rooms," the schedule was readily adapted by all PICU faculty. In these ways, the need to adopt video rounds improved progress in our PFCR more effectively than past efforts.

Specific challenges with telemedicine rounds occurred, some of which have been previously described. Education of trainees became less of a priority during telemedicine rounds, and commonly occurring multidisciplinary discussions on patient assessment and management options were abandoned for more concise plan development, in an attempt to 
maintain efficiency and adhere to the predetermined rounding schedule. However, rounds ended nearly 90 minutes quicker on average, once the process was established. While trainees commonly feel pressured to implement finalized plans immediately following rounds (follow-up with nurses, check orders, contact consultants, and write notes), efforts are underway to prioritize the use of this additional time for education.

The need for protected and managed hardware to ensure patient privacy prevented easy alteration of hardware to improve patient or provider interface and did not allow out-of-hospital family participation. Because we did not have dedicated information technology services support for this inpatient activity, audio-visual issues were addressed by a variety of workarounds. For example, similar to others, we employed a USB "plug and play" speakerphone to enhance audio. ${ }^{12}$ However, we could not directly link this to the PICU iPad, so a team member needed to $\log$ into rounds on his/her personal device. Overall, this slowed improvements in technology, but creative approaches permitted improvements in the ability for the team to hear and see one another.

While family feedback was sought as part of our initial efforts, ongoing efforts seek to formally solicit feedback from family and other team members about the rounding experience to guide ongoing improvement. Our expectation is that some form of socially distanced rounds will continue as the pandemic continues to evolve. We are developing workflows for varying numbers of providers at the bedside based on regional physical-distancing policies. The process has also changed how bedside nurses access the team during rounds when urgent issues arise.

\section{CONCLUSIONS}

The COVID-19 pandemic has forced hospitals to adapt to stay-at-home and social distancing orders, severely compromising the feasibility of the pre-coronavirus rounding process. Hospitals have been required to adapt to this new reality with little guidance in the process. Increasing use of telemedicine applications has been used to help with these requirements, and we herein described our approach to remote telemedicine rounds in the PICU to permit social distancing, while maintaining essential patient communication and family engagement, to help guide others in the process. Despite challenges, telemedicine can facilitate efficient PFCR, while potentially promoting increased time for trainee education and direct patient care at the bedside. The use of available platforms and team-based PI is critical for successful implementation.

Funding Statement: The authors have declared no sources of funding related to this project.

Conflicts of Interest: The authors have declared no conflicts of interest related to this project. The submitting author warrants that he/she is authorized by all coauthors to vouch for the information to be true and correct.

Contributors: All authors participated in the described quality improvement project, writing and critical review of the manuscript, and approved the final version.

\section{References}

1. Control CfD. Coronavirus disease 2019 (COVID-19). 2020. Available from: https:// www.cdc.gov/coronavirus/2019-ncov/ index.html

2. Kates J, Tolbert J. Stay-at-home orders to fight COVID-19 in the United States: The risks of a scattershot approach. 2020. 
Available from: https://www.kff.org/ coronavirus-policy-watch/stay-at-homeorders-to-fight-covid19/

3. Mittal VS, Sigrest T, Ottolini MC, et al. Family-centered rounds on pediatric wards: A PRIS network survey of US and Canadian hospitalists. Pediatrics. 2010;126(1):37-43. https://doi. org/10.1542/peds.2009-2364

4. Lee TS, Kuo MH. Toyota A3 report: A tool for process improvement in healthcare. Stud Health Technol Inform. 2009;143:235-40.

5. Stapleton FB, Hendricks J, Hagan P, DelBeccaro M. Modifying the Toyota Production System for continuous performance improvement in an academic children's hospital. Pediatr Clin North Am. 2009;56(4):799-813. https://doi. org/10.1016/j.pcl.2009.05.015

6. Christoff P. Running PDSA cycles. Curr Probl Pediatr Adolesc Health Care. 2018;48(8):198-201. https://doi. org/10.1016/j.cppeds.2018.08.006

7. Taylor MJ, McNicholas C, Nicolay C, Darzi A, Bell D, Reed JE. Systematic review of the application of the plan-do-study-act method to improve quality in healthcare. BMJ Qual Saf. 2014;23(4):290-8. https:// doi.org/10.1136/bmjqs-2013-001862

8. Sisk B, Alexander J, Bodnar C, et al. Pediatrician attitudes toward and experiences with telehealth use: Results from a national survey. Acad Pediatr. 2020;20(5):628-35. https://doi. org/10.1016/j.acap.2020.05.004

9. American Hospital Association. Telehealth connects patients to vital health care services through videoconferencing, remote monitoring, electronic consults and wireless communications. Chicago, IL. 2019. Available from: https://www. aha.org/system/files/2019-02/fact-sheettelehealth-2-4-19.pdf

10. Badawy SM, Radovic A. Digital approaches to remote pediatric health care delivery during the COVID-19 pandemic: Existing evidence and a call for further research.
JMIR Pediatr Parent. 2020;3(1):e20049. https://doi.org/10.2196/20049

11. Human USDoH, Services. Notification of enforcement discretion for telehealth remote communications during the COVID-19 nationwide public health emergency. Available from: https:/www.hhs.gov/hipaa/ for-professionals/special-topics/emergencypreparedness/notification-enforcementdiscretion-telehealth/index.html

12. Vilendrer S, Patel B, Chadwick W, et al. Rapid deployment of inpatient telemedicine in response to COVID-19 across three health systems. J Am Med Inform Assoc. 2020;27(7):1102-9. https://doi.org/10.1093/ jamia/ocaa077

13. Kaczmarek BF, Trinh QD, Menon M, Rogers CG. Tablet telerounding. Urology. 2012;80(6):1383-8. https://doi. org/10.1016/j.urology.2012.06.060

14. Hron JD, Parsons CR, Williams LA, Harper MB, Bourgeois FC. Rapid implementation of an inpatient telehealth program during the COVID-19 pandemic. Appl Clin Inform. 2020;11(3):452-9. https://doi. org/10.1055/s-0040-1713635

15. Rogers A, Lynch K, Toth H, Weisgerber M. Patient and family centered (Tele)rounds: The use of video conferencing to maintain family and resident involvement in rounds. Acad Pediatr. 2020;20(6):765-6. https://doi. org/10.1016/j.acap.2020.05.026

16. Kuntz JG, Kavalieratos D, Esper GJ, et al. Feasibility and acceptability of inpatient palliative care E-family meetings during COVID-19 pandemic. J Pain Symptom Manage. 2020. https://doi.org/10.1016/j. jpainsymman.2020.06.001

17. Humphreys J, Schoenherr L, Elia G, et al. Rapid implementation of inpatient telepalliative medicine consultations during COVID-19 pandemic. J Pain Symptom Manage. 2020;60(1):e54-9. https://doi. org/10.1016/j.jpainsymman.2020.04.001

18. Yager PH, Cummings BM, Whalen MJ, Noviski N. Nighttime telecommunication between remote staff intensivists and bedside personnel in a pediatric intensive 
care unit: A retrospective study. Crit Care Med. 2012;40(9):2700-3. https://doi. org/10.1097/CCM.0b013e3182591dab

19. Yager PH, Clark M, Cummings BM, Noviski N. Parent participation in pediatric intensive care unit rounds via telemedicine: Feasibility and impact. J Pediatr. 2017;185:181-6.e3. https://doi.org/10.1016/j. jpeds.2017.02.054

20. Berrens ZJ, Gosdin CH, Brady PW, Tegtmeyer K. Efficacy and safety of pediatric critical care physician telemedicine involvement in rapid response team and code response in a satellite facility. Pediatr Crit Care Med. 2019;20(2):172-7. https://doi.org/10.1097/ PCC.0000000000001796

21. Agasthya N, Foo K, Smith T, Slamon N. Creation of a rudimentary electronic pediatric intensive care unit model to explore resident-attending communication. Telemed J E Health. 2020;26(1):101-6. https://doi.org/10.1089/tmj.2018.0190

22. Rea KE, Rao P, Hill E, Saylor KM, Cousino MK. Families' experiences with pediatric family-centered rounds: A systematic review. Pediatrics.
2018;141(3). https://doi.org/10.1542/ peds.2017-1883

23. Smith AC, Thomas E, Snoswell CL, et al. Telehealth for global emergencies: Implications for coronavirus disease 2019 (COVID-19). J Telemed Telecare. 2020;26(5):309-13. https://doi. org/10.1177/1357633X20916567

24. Kipps AK, Albert MS, Bomher S, Cheung S, Feehan S, Kim J. Schedule-based family-centered rounds: A novel approach to achieve high nursing attendance and participation. Pediatr Qual Saf. 2020;5(2):e265. https://doi.org/10.1097/ pq9.0000000000000265

Copyright Ownership: This is an open access article distributed in accordance with the Creative Commons Attribution Non Commercial (CC BY-NC 4.0) license, which permits others to distribute, adapt, enhance this work non-commercially, and license their derivative works on different terms, provided the original work is properly cited and the use is noncommercial. See: http://creativecommons. org/licenses/by-nc/4.0. 


\section{ADDENDUM}

PICU Rounds VidyoConnect Quick Setup

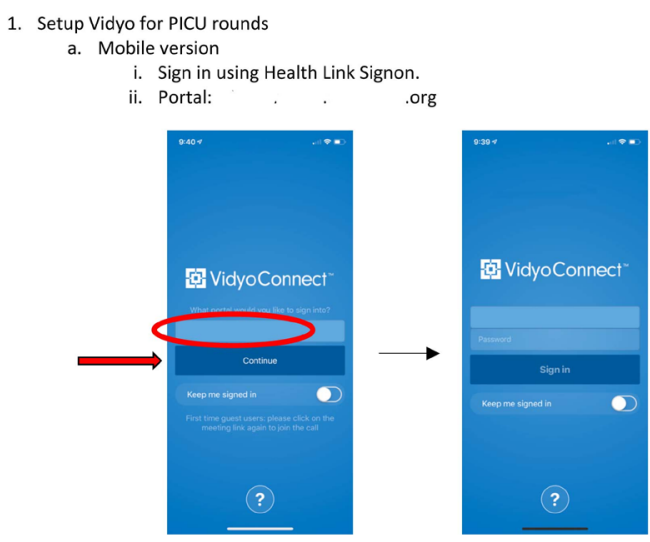

iii. Add PICU Telemed Rooms to list (Search on bottom and type "PICU")

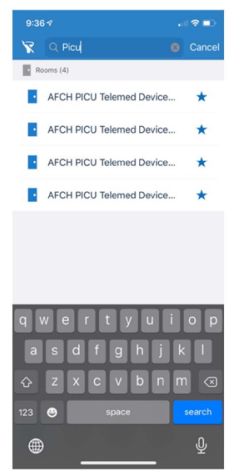

b. Desktop Version (OLD):

i. Sign in using Health Link Signon.

ii. Portal: .org

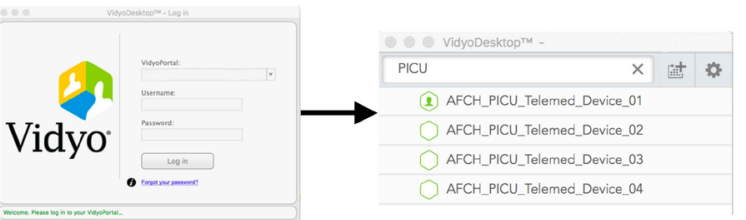

c. Desktop version (VidyoConnect - NEWER)

i. Sign in using Health Link Signon.

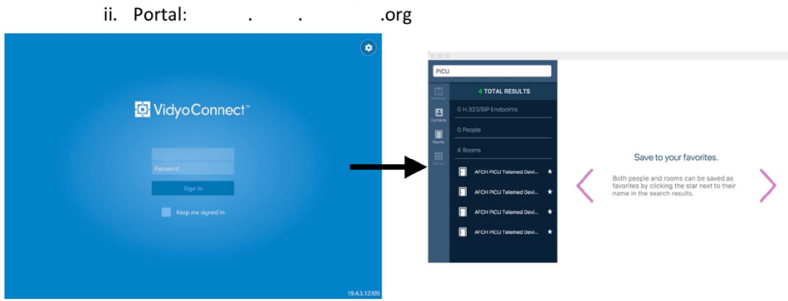

2. Process for rounds:

a. The time for rounds, and the specific PICU telemed room will be posted in the patient's sticky notes and itinerary. Make sure the date of the sticky note is today!

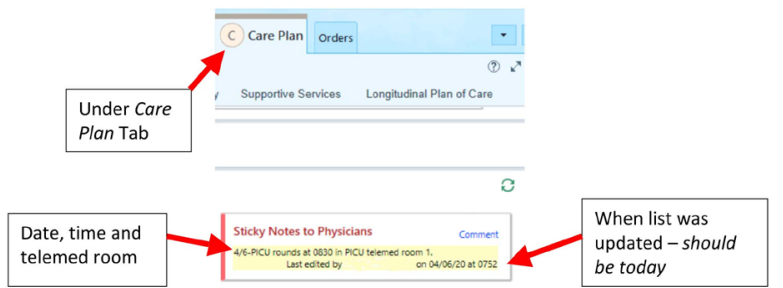

Supplement 1a: Vidyo video conferencing instructions.

$\mathrm{AFCH}=$ American Family Children's Hospital, PICU = pediatric intensive care. 


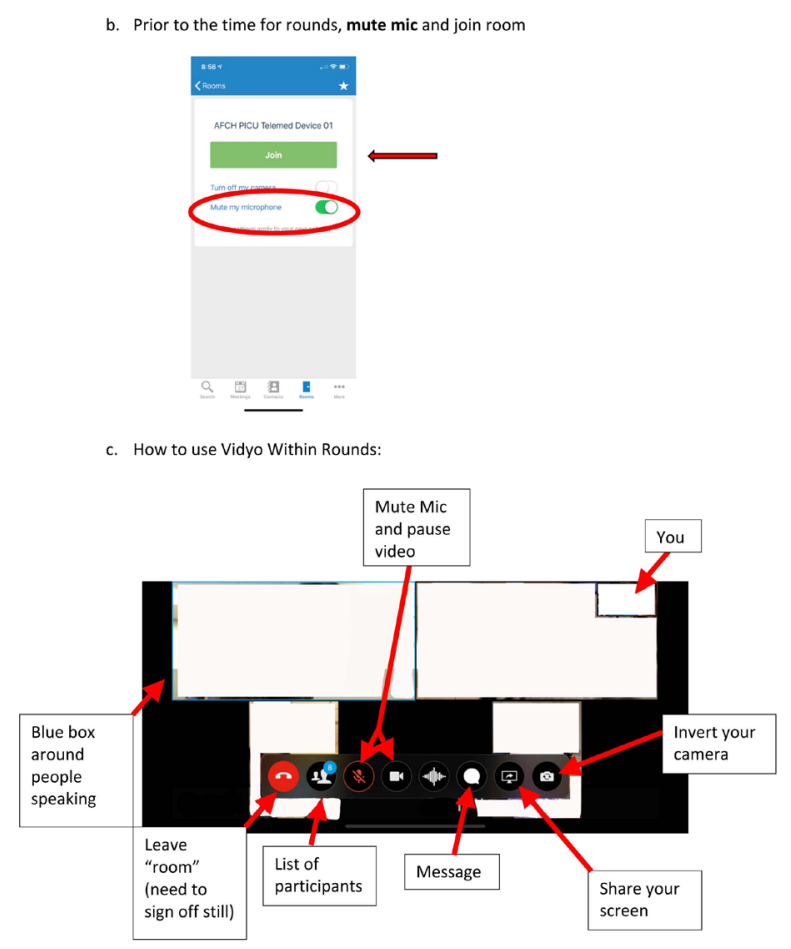

d. The Tool Bar will fade, and can be brought up by touching the screen (on mobile devices) or moving the cursor (on a desktop).

e. Turning your device will change the view from landscape to portrait

f. Portrait View:

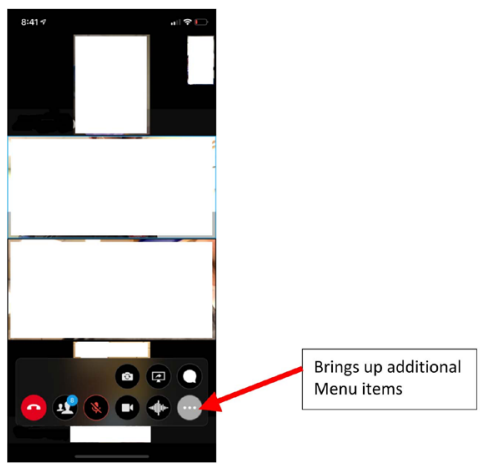

g. Flow of rounds: Rounds will be scripted and each participant will be asked fo their input to prevent confusion and feedback from the mics.

i. It is important to not interrupt as it makes everyone inaudible

Make sure previous patient/nurse have left room - rounds may b

slightly off schedule despite best efforts.

ii. Current nurse will say they're ready to start

iv. "What concerns do family/nurse want to address?"

v. Primary critical care provider will deal with one problem (or system) at a time.

1. Present data

2. Present assessment

3. Present Plan

4. They will ask each relevant team member for each problem, specifically, for input

5. Summarize this problem

vi. Repeat v.1-5 for each problem

vii. CCM lead will let the team know if approaching time limit.

1. If necessary, will schedule more time after rounds.

Supplement 1b: Vidyo video conferencing instructions.

viii. CCM lead summarizes overall plan

ix. CCM lead will ask for input on plan from each individual team member

$x$. CCM lead will ask bedside nurse and family what questions they have

xi. Nurse (and family) sign off

xii. CTL or HUC calls next nurse and consultants for rounds

xiii. Start again at g.i above

h. When you're done in the room

i. "Hang up"

ii. Sign out - Mobile version: under Settings tab
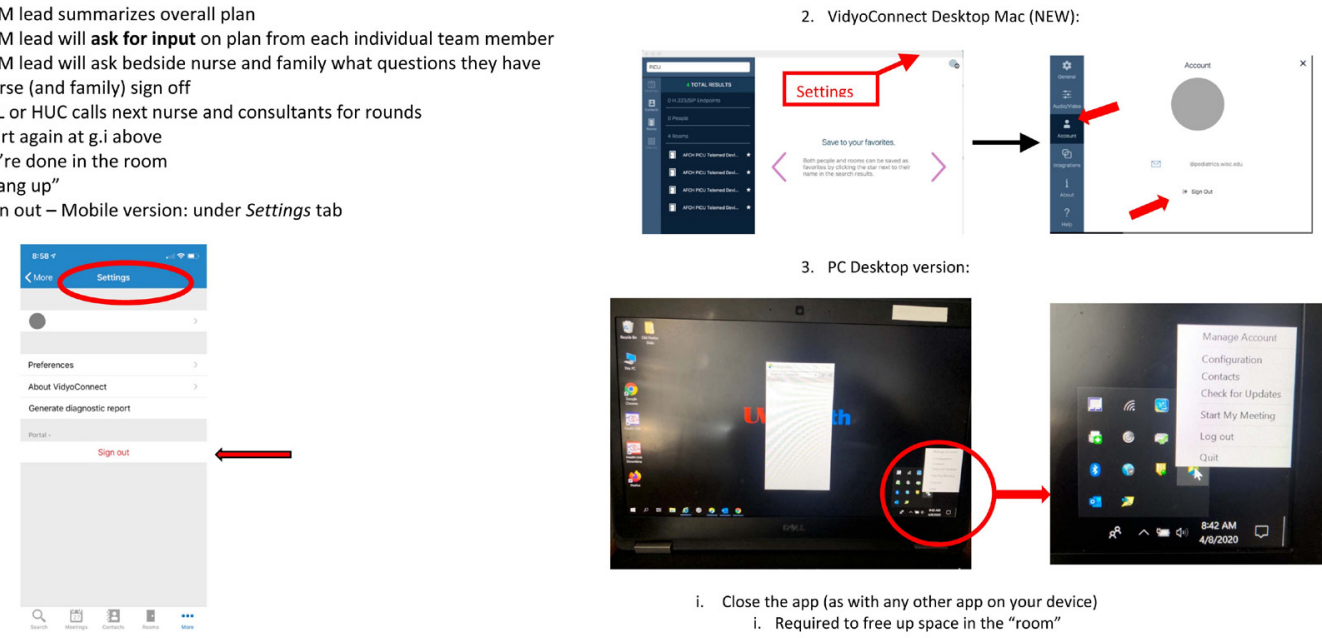

3. PC Desktop version:

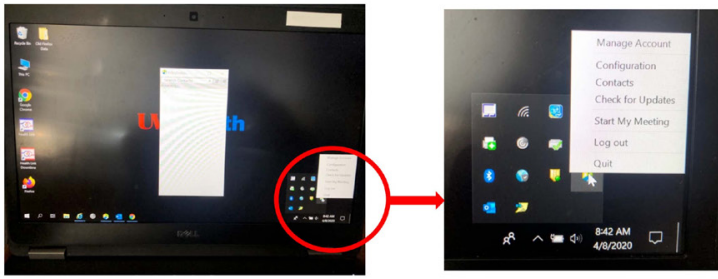

i. Close the app (as with any other app on your device)

Required to free up space in the "room"

1. Mac Desktop version (OID):

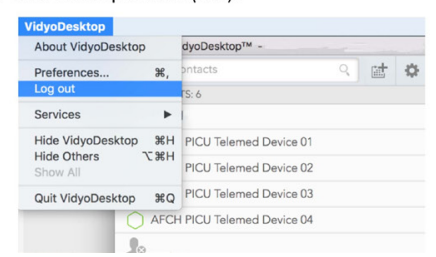

Supplement 1c: Vidyo video conferencing instructions.

$\mathrm{CCM}=$ critical care medicine, $\mathrm{CTL}=$ Care Team Leader, $\mathrm{HUC}=$ Health Unit Coordinator, $\mathrm{PICU}=$ pediatric intensive care. 


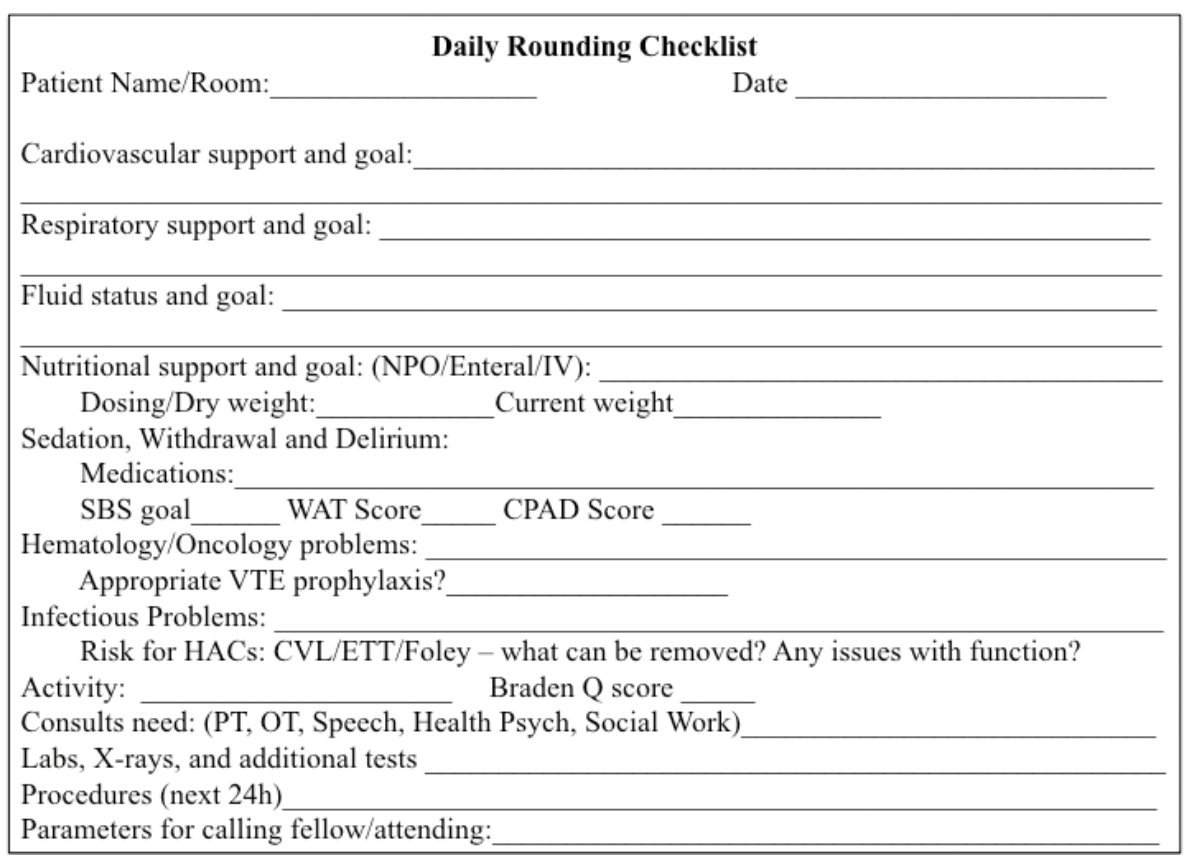

\section{Supplement 2: Pediatric intensive care unit rounding checklist.}

$\mathrm{CPAD}=$ Cognitive Perceptual Assessment for Driving, $\mathrm{CVL}=$ central venous line, $\mathrm{ETT}=$ endotraceal tube, $\mathrm{HAC}=$ hospital acquired conditions, NPO = nothing by mouth, IV = intravenous, $\mathrm{PT}=$ physical therapy, $\mathrm{SBS}=\mathrm{State}$ Behavioral Scale, $\mathrm{VTE}=$ venous thromboembolism, WAT $=$ Withdrawal Assessment Tool. 